\title{
Guidelines for Noninvasive Vascular Laboratory Testing: A Report from the American Society of Echocardiography and the Society of Vascular Medicine and Biology
}

\author{
Represented by Marie Gerhard-Herman, MD, MMSc, Julius M. Gardin, MD, FASE, \\ Michael Jaff, DO, Emile Mohler, MD, Mary Roman, MD, and \\ Tasneem Z. Naqvi, MD, FASE, RVT
}

\section{EXECUTIVE SUMMARY}

Accompanying the rapid growth of interest in percutaneous vascular interventions, there has been increasing interest among cardiologists in performing noninvasive vascular testing using ultrasound. In an attempt to provide recommendations on the best practices in vascular laboratory testing, this report has been prepared by a writing group from the American Society of Echocardiography (ASE) and the Society of Vascular Medicine and Biology. The document summarizes principles integral to vascular duplex ultrasound-including color Doppler, spectral Doppler waveform analysis, power Doppler, and the use of contrast. Appropriate indications and interpretation of carotid artery, renal artery, abdominal aorta, and peripheral artery ultrasound imaging are described. A dedicated section summarizes noninvasive techniques for physiologic vascular testing of the lower extremity arteries-including measurement of segmental pressures and pulse volume plethysmography. The use of exercise testing in the evaluation of peripheral artery disease, ultrasound evaluation of the lower extremities after percutaneous revascularization, and the diagnosis and management of iatrogenic pseudoaneurysm (PSA) is also discussed. A section on the important

From Brigham and Women's Hospital, Boston, MA (M.G-H.); St. John Hospital and Medical Center, Detroit, MI (J.M.G.); Massachusetts General Hospital, Boston, MA (M.J.); University of Pennsylvania Health System, Philadelphia, PA (E.M.); Weill Medical College of Cornell University, New York, NY (M.R.); Cedars Sinai Medical Center, Los Angeles, CA (T.Z.N.).

(C) Copyright 2005 American Society of Echocardiography (ASE). Property of the ASE. Reprint of these documents, beyond single use, is prohibited without prior written authorization of the ASE.

Reprint requests: The American Society of Echocardiography, 1500 Sunday Dr, Suite 102, Raleigh, NC 27607. (919) 864-7754.

J Am Soc Echocardiogr 2006;19:955-972.

0894-7317/\$32.00

Copyright 2006 by the American Society of Echocardiography. doi:10.1016/j.echo.2006.04.019 topic of vascular laboratory accreditation is included. Finally, additional details regarding proper technique for performance of the various vascular tests and procedures are included in the Appendix.

\section{BACKGROUND}

There has been increasing demand for vascular ultrasound training among cardiologists in practice and in training. For example, the recent document on training for cardiology fellows, COCATS-2, has recommended 2 months of dedicated or aggregate "instruction in the noninvasive laboratory" for Level 1 training in vascular ultrasound. ${ }^{1}$ This article will review general principles, indications, and interpretation of noninvasive vascular testing of the carotid arteries, renal arteries, abdominal aorta, and peripheral arteries. Additional details regarding the techniques of performing vascular ultrasound are provided in the Appendix. Another article by this working group, "Clinical Application of Noninvasive Vascular Ultrasound in Cardiovascular Risk Stratification," will review the application of carotid artery (intimal-medial thickness) and brachial artery (flowmediated dilatation) measurements for cardiovascular risk stratification.

\section{INSTRUMENTATION: GENERAL CONSIDERATIONS}

Vascular testing includes duplex ultrasound and physiologic evaluation. Vascular ultrasound tests require a machine equipped with $5-$ to $12-\mathrm{MHz}$ linear-array transducers (for the neck and extremities) and $2.25-$ to $3.5-\mathrm{MHz}$ curved linear- or phased-array transducers (for the abdomen). A vascular software package is required in addition to the appropriate transducers. Duplex scanning refers to an ultrasound scanning procedure recording both gray scale and Doppler information. 


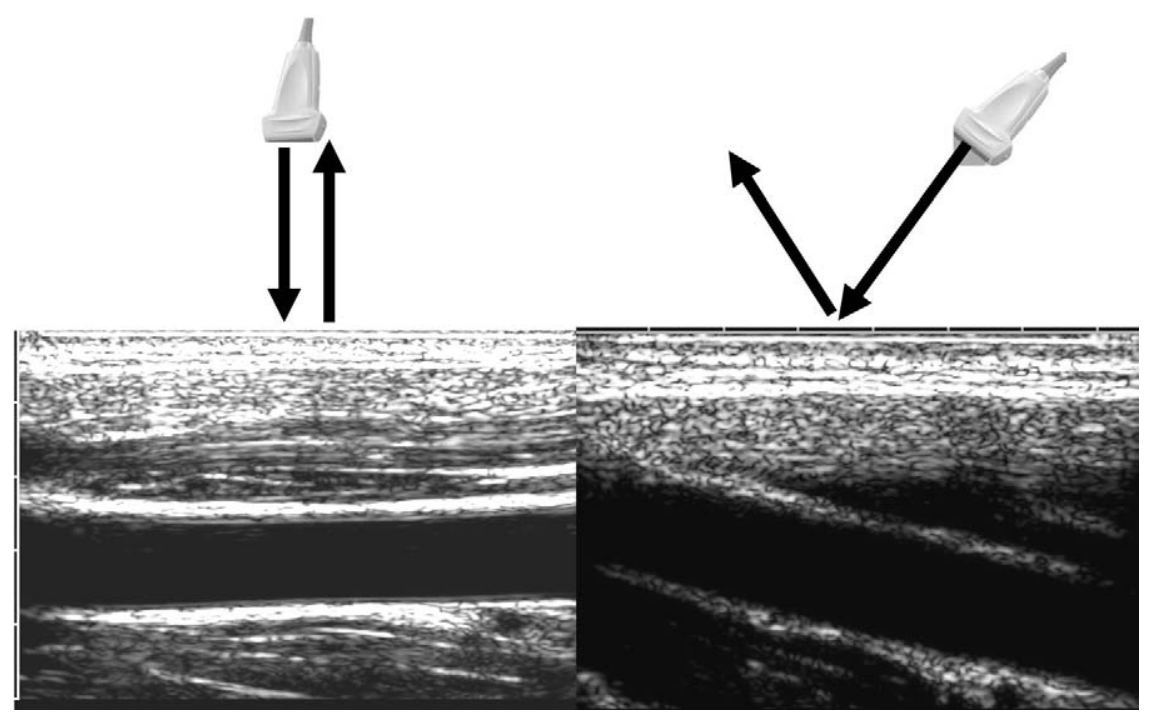

Figure 1 Vessel of interest should be perpendicular to ultrasound beam for B-mode imaging to obtain most distinct echoes. Left, Carotid B-mode image obtained with ultrasound beam perpendicular to vessel wall (arrow) demonstrates trilaminar structure of arterial wall. Right, Wall structure is poorly defined with nonperpendicular angle (arrow).

This includes 2-dimensional structure and motion, Doppler spectrum analysis, and color flow velocity mapping. Carotid arteries, renal arteries, abdominal aorta, and peripheral arteries can be appropriately evaluated using this equipment. Physiologic testing includes segmental pulse volume recording and segmental pressure measurements with cuffs appropriately sized for the lower extremities and a plethysmographic recording device.

\section{DUPLEX: PRINCIPLES APPLICABLE TO ALL VASCULAR TESTING}

The ultrasound beam is directed perpendicular to the surface of interest to obtain the brightest echo with gray-scale imaging and optimal imaging of the artery wall. The perpendicular angle is often readily obtained, as arteries generally are parallel to the surface of the transducer (Figure 1). For the Doppler component of duplex imaging, an angle of 60 degrees between the Doppler insonation beam and the vessel wall should be maintained. This Doppler angle becomes an important consideration when the velocity data are used to classify disease. ${ }^{2}$ Angles above 60 degrees can result in significant overestimation of the velocity and should be avoided. Angles that are not relevant to the vessel wall may misrepresent the true peak velocity $^{3}$ (Figure 2).

\section{Color Doppler}

The pulse repetition frequency scale determines the degree of color saturation and is adjusted so that normal laminar flow appears as a region of homogeneous color. Stenosis results in the production of a high velocity jet and an abrupt change in the color flow pattern. This is identified as either aliasing or desaturation (whitening) of the color display at the site of luminal narrowing. Aliasing occurs when the flow velocity exceeds the Nyquist limit and results in color display of the reverse flow direction (wrap around). The poststenotic region demonstrates a mosaic pattern indicating turbulent flow (Figure 3). Gray-scale settings are adjusted to optimize visualization of intraluminal plaque or thrombus at these sites of abnormal flow. Color Doppler provides additional information used to detect a significant stenosis. Color aliasing, persistence, and bruit all indicate flow disturbance. Color persistence is a continuous flow signal that is color of the forward direction only, in contrast to the alternating color in normal arteries. ${ }^{4}$ Color persistence corresponds to the monophasic spectral Doppler waveform of severe stenosis. A color bruit in the surrounding soft tissue also indicates flow disturbance. This color artifact is attributed to vibration in the surrounding soft tissue in the presence of a high velocity jet. Abnormalities of color flow indicate possible stenosis that is then characterized using pulsed wave Doppler determination of velocities. 

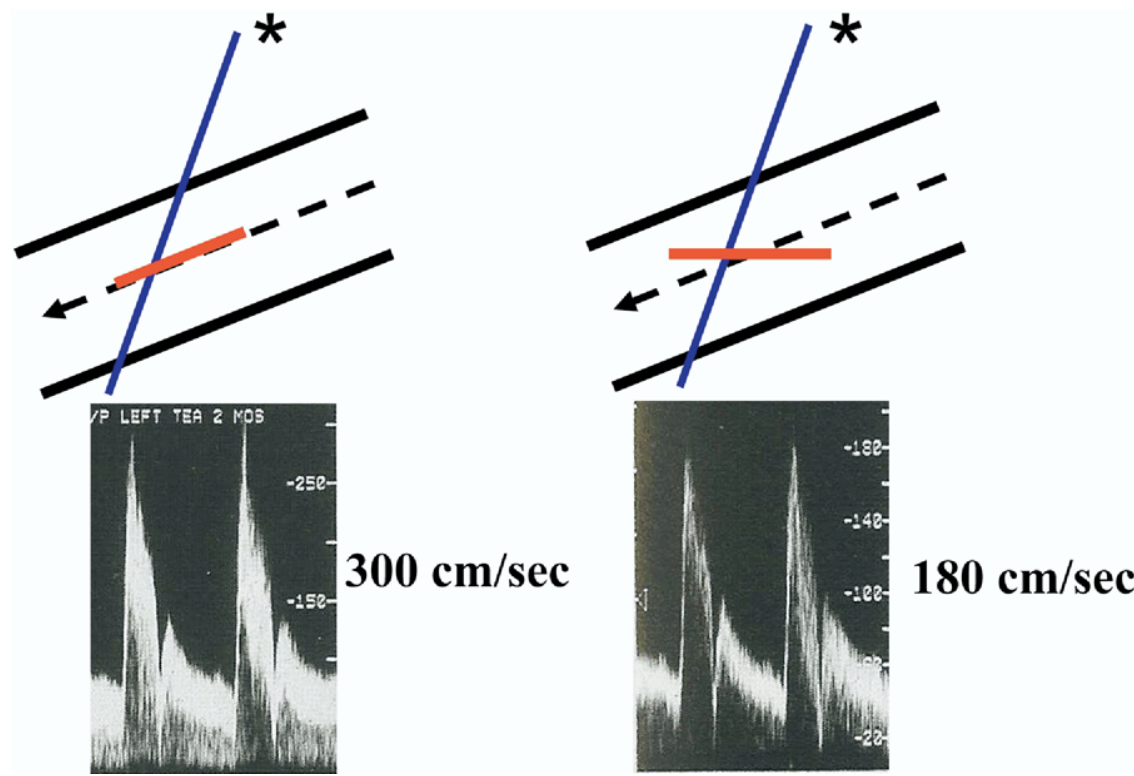

Figure 2 Angle of 60 degrees of Doppler insonation relative to vessel axis provides most accurate Doppler velocities. Angle correction should be used to maintain Doppler angle of 60 degrees or less. Doppler cursor should be parallel to vessel axis in center stream of arterial flow. Left, Appropriate alignment of Doppler beam at 60 degrees to vessel wall with sample volume cursor parallel to vessel axis (imaginary line drawn in center of vessel). Right, Inaccurate Doppler angle. There is misalignment of the cursor not parallel to vessel axis. Pulsed wave Doppler images obtained from same internal carotid artery demonstrates underestimation of peak systolic velocity with inaccurate Doppler angle (right).

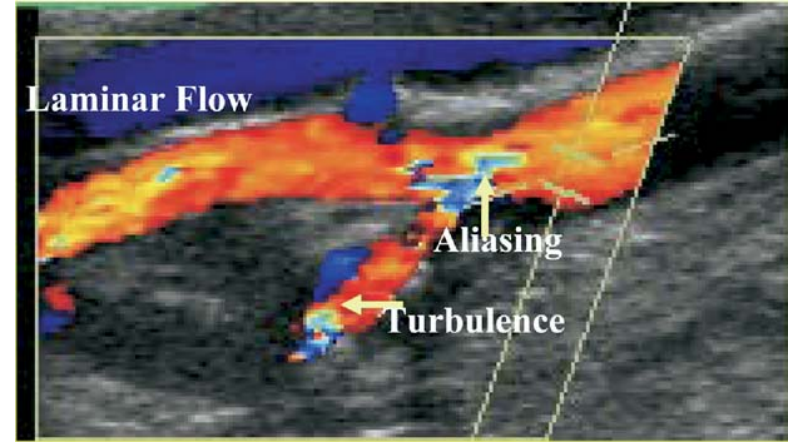

Figure 3 Color Doppler image obtained at carotid bifurcation. Laminar flow in external carotid artery is demonstrated by homogenous color with lightest color toward center of vessel. Aliasing is evident at origin of internal carotid artery (ICA) by abrupt color change from red to blue midstream at origin of ICA where large echolucent plaque is present. Mosaic of color filling remainder of ICA is consistent with poststenotic turbulence.

\section{Spectral Doppler Waveform Analysis}

A normal pulsed wave Doppler waveform is a sharply defined tracing with a narrow Doppler spectrum indicating that blood cells are moving at similar speed throughout the cardiac cycle (Figure 4). Flow becomes turbulent at bifurcations and luminal narrowings causing spectral broadening of Doppler waveform, with filling in of the low velocity region in the spectral waveform as the blood cells move at a wide range of velocities (Figure 4). The normal peripheral artery waveform is triphasic (Figure 4). The first component is the consequence of initial forward flow during systole, and results in peak systolic velocity (PSV) measurements that are typically less than 125 $\mathrm{cm} / \mathrm{s}^{5}$ for each arterial segment. There is early diastolic flow reversal in the second phase of the waveform as left ventricular pressure decreases before aortic valve closure. In late diastole, there is a small amount of forward flow that reflects elastic recoil of vessel walls. This diastolic component is absent in stiff atherosclerotic vessels. Waveform shape is also characterized as high resistance (eg, normal peripheral arterial waveform), or low resistance (eg, normal internal carotid artery [ICA] waveform) (Figure 4). The amount of flow during diastole is determined by the degree of dilation in the distal resistance arterioles.

\section{Power Doppler}

Power (or energy) Doppler is a technique that displays the total strength (amplitude) of the returning Doppler signal without distinguishing direction. ${ }^{6}$ Sensitivity is increased by a factor of 3 to 5 

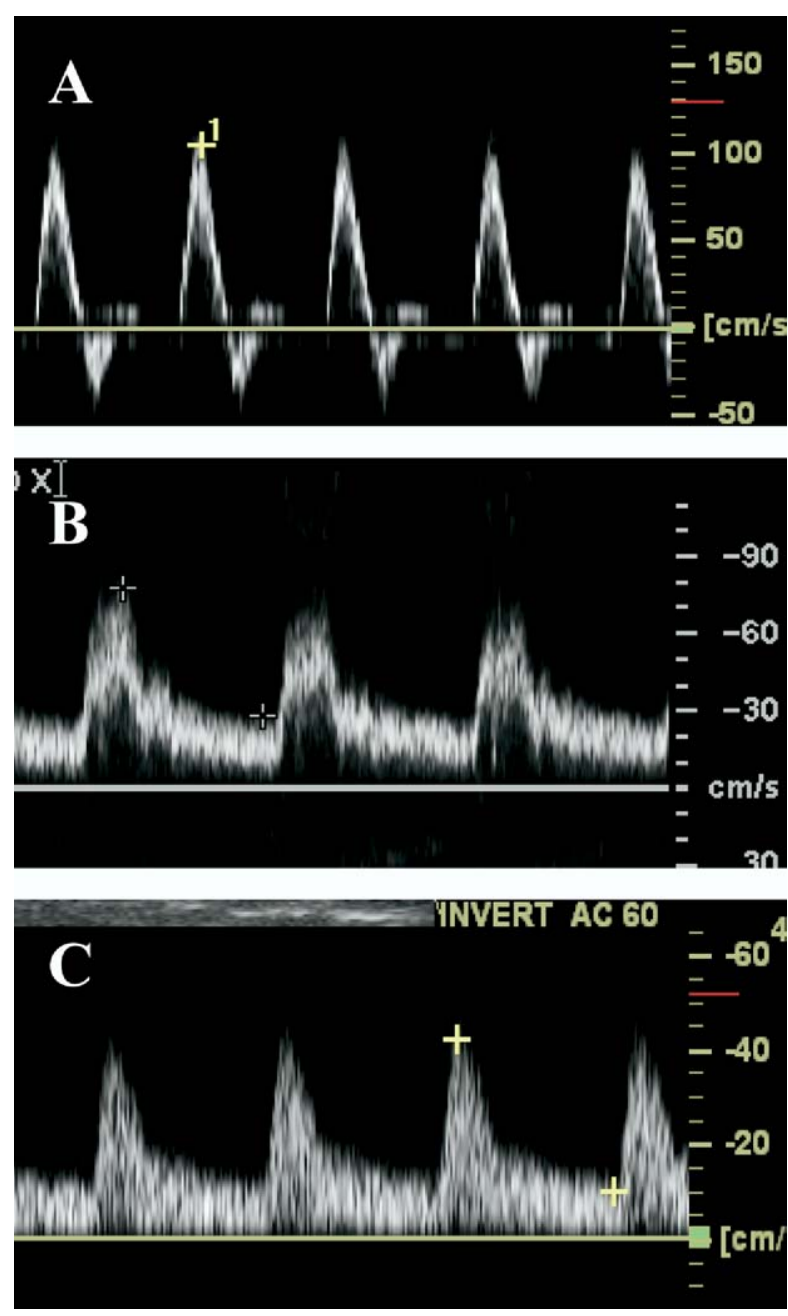

Figure 4 Spectral Doppler waveforms. A, Normal laminar flow with narrow range of velocities throughout cardiac cycle. Cross is placed above clear spectral window (or envelope). B, Biphasic Doppler waveform with loss of reverse diastolic flow and mild widening of spectral envelope (spectral broadening). C, Turbulent flow with filling in of spectral window (spectral broadening) and low peak systolic velocities.

times $^{6}$ with power Doppler compared with color flow Doppler. Power Doppler can, therefore, identify very slow flow that may not be detected by color flow Doppler. Power Doppler is less angle dependent than is color Doppler and it improves delineation of the lumen. ${ }^{7}$ Power Doppler is used to differentiate high-grade stenosis from occlusion, to detect collateral vessels, and to identify small vessel disease.

\section{Assessment of Arterial Stenosis}

Doppler velocity is the main tool used to evaluate stenosis severity. Characteristic duplex ultrasound features of stenosis include elevated velocities, color disturbance, spectral broadening, and poststenotic waveforms (Table 1). If no poststenotic turbulence can be identified, inappropriate angle alignment or a tortuous vessel should be suggested as a cause of artifactually high velocities.

\section{USE OF CONTRAST IN VASCULAR IMAGING}

Ultrasonographic assessment of peripheral vascular disease is largely dependent on the functioning of the equipment and the skill of the operator. ${ }^{8}$ The addition of duplex color Doppler techniques has allowed for improved identification of the anatomy of peripheral vascular disorders, including the location, length, and presence of stenosis or occlusion; development of collateral vessels; and areas of reconstitution. Ultrasound contrast agents also appear useful in enhancing suboptimal images and improving arterial diagnosis in areas where calcification in the vessel wall obscures the view of the lumen and the ability to determine velocity. ${ }^{3}$ Contrast agents have been shown investigationally to better outline the lumen of the carotid arteries and facilitate measurement of intimal-medial thickness, and to help in outlining plaque morphology, and in differentiating between occlusion and high-grade stenosis. Contrast enhancement of the renal vasculature has been reported to be useful in cases where multiple main renal arteries are present. ${ }^{3,9}$ Several largescale studies have found improvement in peripheral artery diagnosis using contrast after suboptimal baseline ultrasound scans. ${ }^{10}$ Contrast appears to have use in improving images of vessels difficult to adequately capture using traditional ultrasound techniques, such as the iliac arteries, the superficial artery in the adductor canal, the trifurcation vessels, and the plantar arteries. Contrast enhancement may also be useful in differentiating between patent and nonpatent vessels in patients with conditions that interfere with ultrasound scanning (ie, obesity, edema, dense calcification). ${ }^{11}$ Nonetheless, it should be emphasized that despite these reports documenting the efficacy of contrast agents in enhancing vascular ultrasound imaging, they have not received US Food and Drug Administration approval for all these indications and, hence, this application should still be, at present, considered experimental.

\section{CAROTID ARTERY ULTRASOUND}

The goal of noninvasive ultrasound testing for carotid disease is to distinguish normal from diseased vessels, to classify a wide range of disease states, to assess the cerebral collateral circulation, 
Table 1 Duplex evidence of arterial stenosis

- Elevated velocities: diagnostic criteria use peak systolic velocity (eg, $>125 \mathrm{~cm} / \mathrm{s})$, ratios of distal to proximal sequential peak systolic velocities (eg, 2:1), and elevated end-diastolic velocity, supportive criteria include aliasing of color Doppler signal

- Diameter reduction: transverse or longitudinal measurements indicating reduction in luminal diameter are supportive, not diagnostic

- Spectral broadening or color mosaic pattern: the presence of turbulent flow is supportive, not diagnostic; it is most prominent just distal to significant stenosis

- Color bruit, color persistence: color bruit, providing evidence of vibration in the tissue surrounding arterial narrowing, is supportive, not diagnostic; continuous forward flow, or persistence, is supportive evidence of arterial stenosis

Table 2 Indications for carotid artery ultrasound

- Cervical bruits

- Amaurosis fugax

- Hemispheric stroke

- Focal cerebral or ocular transient ischemic attacks (which demonstrate localizing symptoms, such as weakness of one side of the face, slurred speech, weakness of a limb, retinal or hemispheric visual field deficits)

- Drop attacks or syncope (rare indications primarily seen in vertebrovascular insufficiency or bilateral carotid artery disease)

- Vasculitis involving extracranial arteries

- Pulsatile mass in the neck

- Trauma to neck

- Follow-up of carotid artery atherosclerosis not requiring revascularization

- Follow-up surveillance after carotid revascularization, a baseline ultrasound is recommended within 30 days after carotid stenting

and to do so in a safe and cost-effective manner. The primary aim is to identify patients who are at risk for stroke and who may require specific treatment. A secondary aim is to document progressive or recurrent disease in patients already known to be at risk. Appropriate indications for carotid artery testing are listed in Table $2 .^{12-15}$

\section{Interpretation}

Duplex imaging should include, at a minimum, common carotid artery (CCA), ICA, external carotid artery, and vertebral artery. The interpretation of the spectral waveforms is based on parameters such as PSV, end-diastolic velocity, and the extent of spectral broadening (Figure 5). Individual vascular laboratories must validate their own results against a suitable gold standard (eg, arteriography). Several velocity criteria used to detect presence and severity of carotid artery disease have been published. ${ }^{16}$ Table 3 summarizes useful absolute velocities and velocity ratios to diagnose significant ICA stenosis. ${ }^{16}$ When all categories of carotid disease are considered, criteria distinguishing between normal and diseased ICA have a specificity of $84 \%$ and a sensitivity of $99 \%$ when compared with angiography. ${ }^{17}$ The accuracy for detecting $50 \%$ to $99 \%$ diameter stenosis is $93 \%$. The agreement with angiography is excellent for

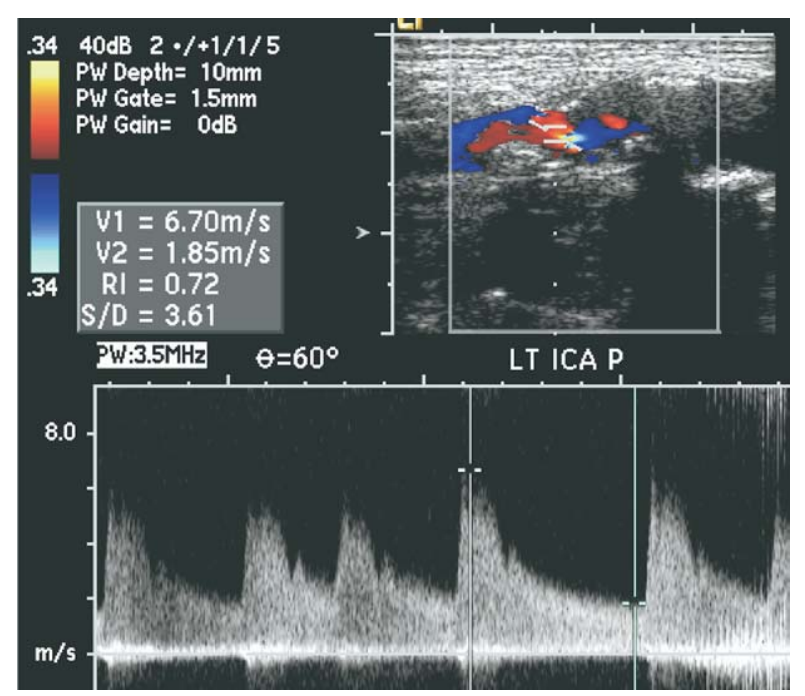

Figure 5 Duplex evidence of internal carotid artery (ICA) stenosis. Duplex image, sample volume cursor parallel to artery wall, at site of color aliasing and luminal narrowing. Spectral Doppler, high peak systolic velocity $(670 \mathrm{~cm} / \mathrm{s})$, high end-diastolic velocity $(185 \mathrm{~cm} / \mathrm{s})$, and spectral broadening (turbulence). These findings indicate severe $(80 \%$ 99\%) ICA stenosis.

classification of lesions that result in greater than $50 \%$ diameter reduction. ${ }^{18}$ Experience with duplex scanning in patients undergoing carotid endarterectomy indicates that the results of arteriography rarely altered the clinical treatment plan when a technically adequate duplex scan showed an $80 \%$ to $99 \%$ stenosis in an asymptomatic patient, or ipsilateral $50 \%$ to $99 \%$ stenosis in a patient with hemispheric neurologic symptoms. ${ }^{19,20}$

Absolute velocity criteria by duplex ultrasound may be less reliable than change in velocity criteria over time to diagnose recurrent stenosis after carotid artery stenting. A thumping sound may be encountered at the origin of the occluded ICA as a result of flow striking the occlusion followed by flow reversal. Stenosis proximal to the imaged segment is suggested by parvus et tardus waveforms. Diagnosis criteria for stenosis in the CCA are less extensively described. A doubling of PSV from proximal to distal sample indicates greater than $50 \%$ stenosis. A parvus et tardus waveform in 
Table 3 Criteria for classification of internal carotid artery disease by duplex scanning with spectral waveform analysis of pulsed Doppler signals

\begin{tabular}{lcccc}
\hline Degree of stenosis, $\%$ & ICA/PSV, $\mathbf{c m} / \mathbf{s}$ & Plaque estimate, $\%$ & ICA EDV, $\mathbf{~ c m} / \mathbf{s}$ & ICA CCA PSV ratio \\
\hline Normal & $<125$ & 0 & $<40$ & $<2$ \\
$<50$ & $<125$ & $<50$ & $<40$ & $<2$ \\
$50-69$ & $125-230$ & $>50$ & $40-100$ & $2-4$ \\
$>70$ & $>230$ & $>50$ & $>100$ & $>4$ \\
Subtotal occlusion & Variable & $>50$ Narrow lumen & $>0$ & Variable \\
Total occlusion & 0 & $>50$ & 0 & $<1$
\end{tabular}

$C C A$, Common carotid artery; $E D V$, end-diastolic velocity; ICA, internal carotid artery; PSV, peak systolic velocity.

Table 4 Differentiation of internal and external carotid arteries

\begin{tabular}{ll}
\hline Internal carotid artery & \multicolumn{1}{c}{ External carotid artery } \\
- Usually larger & - Usually smaller \\
- Usually lateral and posterior & - Usually medial and anterior \\
- Usually incorporates carotid & - Usually does not incorporate \\
bulb & bulb \\
- No branches in the neck & - Eight branches in the neck \\
- Low resistance spectral & - High resistance spectral wave- \\
waveform & form at rest \\
- Usually no oscillations in & - Visible and audible oscillations \\
Doppler on temporal tap & on Doppler signal waveform \\
test & on temporal tap test
\end{tabular}

the CCA again suggests there is stenosis proximal to the imaged region. Interpretation should comment on the direction of vertebral artery flow, forward (toward the brain) or reverse (away from the brain). In addition, the vertebral artery waveform should be described as normal (low resistance) or abnormal (biphasic or high resistance). Correct determination of ICA versus external carotid artery is essential for interpretation of study results (Table 4 ).

\section{Limitations and Pitfalls of Carotid Duplex Ultrasound}

Disease may be underestimated in the presence of long smooth plaque that does not have the accelerated turbulent flow patterns associated with hemodynamically significant lesions. High and low cardiac output can affect PSV. In the setting of markedly abnormal cardiac output, the ICA/CCA PSV ratio should be the primary diagnostic criteria for ICA stenosis. Contralateral ICA occlusion may result in overestimation of stenosis in the ipsilateral carotid artery, ${ }^{8}$ due to compensatory increase in peak systolic velocity.

\section{RENAL ARTERY DUPLEX ULTRASOUND}

Atherosclerotic renal artery stenosis has become increasingly recognized as a contributing factor to resistant hypertension, ${ }^{21}$ and may promote deterioration in renal function. Patients with severe bilateral renal artery stenosis, or stenosis to a solitary functioning kidney, are at risk for the development of end-stage renal disease. ${ }^{22}$ Longterm survival of patients with atherosclerotic renal artery stenosis requiring dialysis support is dismal. ${ }^{23}$ Although a number of noninvasive methods of diagnosis in renal artery stenosis have been proposed, none have obviated the role of the gold standard, renal arteriography. Each screening test has significant limitations that prevent widespread acceptance.

\section{Indications, Performance, and Interpretation}

Duplex ultrasonography is the ideal method of determining the adequacy of renal artery revascularization (Table 5). ${ }^{25}$ Duplex ultrasonography is helpful in detecting important areas of restenosis after endovascular therapy (percutaneous angioplasty with stent deployment). ${ }^{26}$ The renal duplex examination includes spectral Doppler velocities from the renal arteries, renal parenchyma, and abdominal aorta. PSV and peak end-diastolic velocities obtained in branches of the renal artery at the level of the medulla are used to calculate the renal resistive index, a value reflecting the health of the renal parenchyma itself (Table 6). In addition, the examination should define the pole-to-pole length of each kidney. Figure 6 illustrates duplex findings of renal artery stenosis.

\section{ULTRASOUND IMAGING FOR ABDOMINAL AORTIC ANEURYSM}

\section{Indications}

Ultrasound imaging is highly sensitive for assessing and following up abdominal aneurysms. ${ }^{27,28} \mathrm{~A}$ family history of an abdominal aneurysm has been reported to increase the risk of developing this condition 4-fold. In addition, if an aneurysm is found in one vascular territory, such as the popliteal artery, there is an increased risk of an aneurysm in the aorta. Major indications for assessment of abdominal aortic aneurysm with ultrasound imaging are included in Table 7 . 
Table 5 Indications for renal duplex ultrasound ${ }^{24}$

- Sudden exacerbation of previously well-controlled hypertension

- New onset hypertension at a young age

- Malignant hypertension

- Unexplained azotemia

- Hypertension and aortoiliac or infrainguinal atherosclerosis

- Azotemia after administration of an angiotensin-converting enzyme inhibitor

- An atrophic kidney

- Recurrent flash pulmonary edema without cardiac explanation

- Evaluation of adequacy of renal artery revascularization

- Detection of restenosis after endovascular therapy

\section{Interpretation}

A normal diameter of the abdominal aorta is approximately $2.0 \mathrm{~cm}$ (range: $1.4-3.0 \mathrm{~cm}$ ) in most individuals (Table 8). A mildly dilated abdominal aorta is described as ectatic, whereas it is reported as aneurysmal when the diameter is greater than $3.0 \mathrm{~cm} .^{28,29}$ Abdominal aortic aneurysms are described as saccular (ie, having a baglike structure protruding asymmetrically from the aorta); fusiform (ie, spindle-shaped and tapering from the middle toward each end); or cylindric. The majority of abdominal aortic aneurysms are fusiform in shape, located below the renal arteries, and they may involve one or both of the iliac arteries. Atherosclerotic changes and/or mural thrombus can line the aneurysmal sac. Dissection has been reported with abdominal aortic aneurysm, but is not common. The typical growth rate reported in the literature of abdominal aortic aneurysms measuring 3 to $5.9 \mathrm{~cm}$ is approximately 0.3 to $0.4 \mathrm{~cm}$ per year. ${ }^{30}$ However, larger aneurysms may progress more quickly than others. Aneurysms repaired by endografts and endovascular stents have unique ultrasound characteristics. Thrombus develops in the aneurysm outside of the endograft. Over time, the maximal diameter of the aneurysm sac surrounding an endograft is expected to decrease.

\section{NONINVASIVE PHYSIOLOGIC VASCULAR TESTING OF THE LOWER EXTREMITY ARTERIES}

The goals of noninvasive testing for peripheral arterial disease are to confirm a clinical diagnosis and further define the level and extent of obstruction. A variety of algorithms are used to noninvasively diagnose peripheral arterial disease in the vascular laboratory. Some of these include segmental limb pressures with pulse volume plethysmography, exercise treadmill testing, and arterial ultrasonography.
Table 6 Diagnostic criteria for significant renal artery stenosis

Renal artery to aorta peak systolic velocity ratio is $>3.5$ PSV $>200 \mathrm{~cm} / \mathrm{s}$ with evidence of poststenotic turbulence $\mathrm{EDV}>150 \mathrm{~cm} / \mathrm{s}$ ( $>80 \%$ renal artery stenosis)

$\mathrm{RI}>0.8$ (used to predict response of blood pressure, renal function, to renal revascularization)

An occluded renal artery demonstrates no flow in the affected vessel

$E D V$, End-diastolic velocity; $P S V$, peak systolic velocity; $R I$, resistive index $(1-[\mathrm{EDV} /$ maximum systolic velocity $] \times 100)$.

The major indications for assessment of peripheral arterial disease with noninvasive testing are summarized in Table 9.

\section{Segmental Limb Pressure Interpretation}

Systolic intravascular pressures increase slightly from the femoral to the tibial level so that intraarterial measurement of pressures would typically demonstrate a higher pressure in the tibial artery than in the femoral artery. When the 4-cuff method is used with relatively narrow cuffs, a pressure artifact is introduced into the measurement, accounting for the gradual increase in measured pressure in ascending levels of the leg. In healthy individuals, the high thigh pressure typically exceeds the ankle pressure. ${ }^{31}$ Thus, a thigh/ brachial index of 1.1 or greater is indicative of normal hemodynamics and an index of less than 0.9 is an indicator of peripheral arterial disease (Table 10). Of note, when the high thigh pressure is low compared with the brachial artery pressure, the level of obstruction may be proximal to the cuff or beneath the cuff. Thus, the site of obstruction could be in the aorta, iliac artery, common femoral artery, or proximal superficial femoral artery. When the inflow hemodynamics are abnormal bilaterally, the site of obstruction may be the aortoiliac region. However, if the low pressures are unilateral, then only an ipsilateral iliac or common femoral stenosis is inferred and an abnormality of the aortic segment should not be included in the interpretation. Segmental limb pressures are compared with adjacent ipsilateral segments, contralateral-paired segment, and greater of the two brachial systolic pressures. A reduction of $20 \mathrm{~mm} \mathrm{Hg}$ or greater in pressures is considered significant if such a gradient is present either between segments along the same leg or when compared with the same level of the opposite leg. ${ }^{32}$

\section{Pulse Volume Plethysmography Interpretation}

Segmental limb plethysmographic waveform analysis is based on evaluation of waveform shape and signal amplitude. Standardized criteria relating waveform changes to anatomic site and hemodynamic 

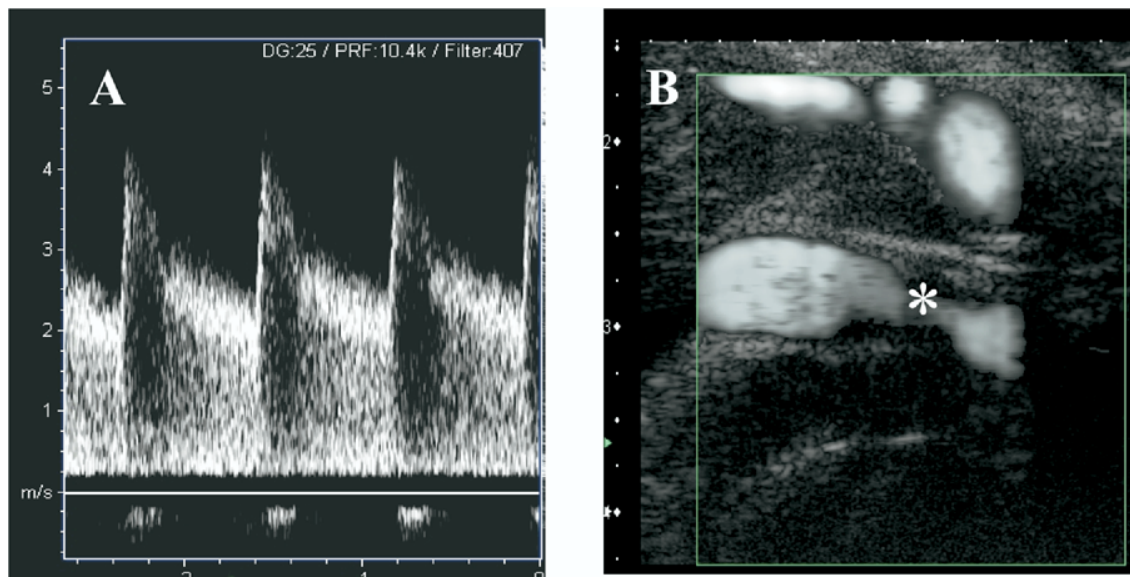

Figure 6 A, Renal artery stenosis. Doppler spectral waveform demonstrates turbulence with marked elevation in peak systolic and end-diastolic velocities. B, Power Doppler indicating luminal narrowing $($ * at site of stenosis

Table 7 Indications for abdominal aorta ultrasound

- Abdominal pain

- Pulsatile and enlarged aorta on physical examination

- Hemodynamic compromise suggestive of a ruptured aneurysm

- An immediate family member with a history of abdominal aortic aneurysm

- An aneurysm found in another vascular territory

- Follow-up of aortic endograft

Table 8 Diagnostic criteria for abdominal aortic aneurysm and endoleak

- Aneurysm: diameter $>3.0 \mathrm{~cm}$

- Endoleak: flow outside of the aortic endograft, and within the aneurysm sac

- Dissection: true and false lumen present

severity of disease are used in diagnostic interpretation. Pulse volume recordings are typically performed by injecting a standard volume of air into pneumatic cuffs. Older machines adjusted for the amount of air in the cuff so that a precise comparison could be made between legs or at various levels along the extremities. Some newer machines, however, do not calibrate the amount of air in the cuff; thus, caution is advised in interpreting the amplitude of the pulse volume recording in comparison with the other extremity or at various levels. The volume of air injected into the cuff is enough to occlude the venous circulation but does not occlude the arterial circulation.

Volume changes in the limb segment below the cuff are translated into a pulsatile pressure, which is detected by a transducer and then displayed by a pressure pulse contour. A normal pulse volume recording, similar to the arterial waveform, is composed of a systolic upstroke with a sharp systolic peak followed
Table 9 Indications for noninvasive physiologic testing

- Exercise-related limb pain (claudication symptoms)

- Limb pain at rest

- Extremity ulcer/gangrene

- Assessment of healing potential

- Absent peripheral pulses

- Digital cyanosis

- Cold sensitivity

- Arterial trauma and aneurysms

- Abnormal ABI

$A B I$, Ratio of ankle-to-brachial arterial systolic blood pressure. Follow-up evaluation is warranted for graft surveillance, worsening symptoms of claudication, and assessment of revascularization therapy.

Table 10 Criteria for abnormal segmental pressure study

\begin{tabular}{ll}
\hline Level of disease & $\begin{array}{c}\text { Findings } \\
\text { High thigh/brachial index } \\
<0.9 \text { bilaterally } \\
\text { High thigh/brachial index } \\
\text { Iliac }\end{array}$ \\
SFA disease & $\begin{array}{c}\text { Gradient between high } \\
\text { and low thigh cuffs }\end{array}$ \\
Distal SFA/popliteal & Gradient between thigh \\
& cuff and calf cuff \\
Infrapopliteal & Gradient between calf and \\
& ankle cuffs
\end{tabular}

Pressure gradient between $20-30 \mathrm{~mm} \mathrm{Hg}$ is borderline, $\geq 30 \mathrm{~mm}$ $\mathrm{Hg}$ is abnormal

$S F A$, Superficial femoral artery.

by a downstroke that contains a prominent dicrotic notch. If a hemodynamically significant stenosis is present, dissipation of energy occurs because of arterial narrowing; this is reflected in a change in the pulse volume recording contour, indicating a proximal arterial obstruction. The amount of variation in the pulse volume recording contour is reflective of disease severity, as shown in Table 11. 
Table 11 Pulse volume plethysmography: Pulse volume recording contour with increasing vascular disease severity

- Sharp upstroke
- Scooped or flat interval between peaks
- Possible dicrotic notch
Mildly abnormal
- Sharp upstroke
- No flat period or scooping between peaks
- No dicrotic notch
- Foderately abnormal
- Equal upslope and downslope time
- No dicrotic notch

\section{Limitations of Segmental Pressure} Measurement and Pulse Volume Recording

Evaluation of segmental pressures and volume plethysmography are an indirect examination, based on which of the anatomic locations of lesions is inferred. Multiple stenoses located at or above the level of the pneumatic cuff may not be distinguished. For example, a decreased thigh pressure may be caused by obstruction of the aortoiliac segment, or common femoral, or combined superficial femoral artery and profundus femoris artery disease.

\section{EXERCISE TESTING FOR PERIPHERAL ARTERIAL DISEASE}

A peripheral arterial lesion may be revealed with measurement of lower extremity pressures after exercise (Tables 12 and 13).

Contraindications to exercise treadmill testing of the lower extremities include: rest pain, noncompressible vessels on a resting study, acute deep venous thrombosis, shortness of breath at rest or with minimal exertion, uncontrolled angina, or a physical disability that limits patient's ability to ambulate on a treadmill. The ankle pressure should increase with exercise as the brachial pressure increases.

\section{PERIPHERAL ARTERIAL ULTRASOUND}

The Duplex ultrasound study is tailored to individual requirements and can be limited to a given arterial segment, or extended to evaluate both limbs in their entirety (Table 14). The goal of the examination is to elucidate the location and severity of extremity arterial stenoses. It can be helpful particularly when planning therapy for known peripheral arterial occlusive disease.

\section{Interpretation}

Peripheral arterial stenosis is characterized using pulsed wave Doppler evaluation. The diagnostic criteria use step-up in PSV ratios from proximal to 
Table 12 Indications for stress testing of the lower extremities ${ }^{33}, 34$

- Normal resting lower extremity arterial segmental limb pressures and pulse volume recordings at rest, in the context of a history of intermittent claudication

- Resting lower extremity segmental limb pressures and pulse volume recording amplitudes mildly abnormal at rest (ie, ABI $>0.80$ but $<0.96$ ) in a patient with a history of intermittent claudication

$A B I$, Ankle-brachial index.

Table 13 Interpretation of postexercise ankle-brachial index

\begin{tabular}{l} 
- ABI $<0.90$ at 1 minute after exercise indicates hemodynami- \\
cally significant PAD \\
\hline
\end{tabular}

$A B I$, Ankle-brachial index; $P A D$, peripheral arterial disease.

distal artery and careful waveform analysis (Table 15). Pulsed Doppler interrogation at the level of a stenosis reveals a PSV double that of the velocity in the proximal segment. There is spectral broadening and forward flow throughout the cardiac cycle in severe stenosis. An occlusion is present when there is no flow within an arterial segment. High-resistance waveforms are present in the artery proximal to the occlusion if there are no collateral vessels. Continuous forward diastolic flow is present in the proximal artery if dilated high capacitance collaterals are present. The artery that reconstitutes distal to a high-grade stenosis will have a characteristic poststenotic parvus et tardus waveform.

Doppler examination can accurately diagnose peripheral arterial disease. ${ }^{35,36}$ Compared with the gold standard of arteriography, duplex Doppler evaluation used to detect significant stenoses in patients with proximal lower extremity arterial disease demonstrates a high sensitivity (82\%) and specificity (92\%). Use of color and pulsed wave Doppler $^{36,37}$ increases the sensitivity $(87 \%-88 \%)$ and specificity (95\%-99\%) of stenosis identification. The PSV ratio between the stenosis and the immediately proximal artery segment classifies peripheral arterial stenoses better than absolute PSV measurements. For example, PSV ratios of 2 and 7 correspond to stenoses greater than 50\% and greater than $90 \%$, respectively. ${ }^{38}$ There is a wide range of PSV measurements obtained in the lower extremities of normal and abnormal cases. There is a greater correlation between PSV ratio and stenosis than between absolute PSV and stenosis.

\section{PERIPHERAL ARTERIAL BYPASS GRAFT ULTRASOUND}

Graft surveillance has proven quite useful in efforts to preserve the patency of bypass grafts. Infrainguinal bypass graft 5-year primary patency rate ranges from $60 \%$ to $85 \%{ }^{39}$ Surgical revision of
Table 14 Indications for peripheral arterial ultrasound

Claudication

Leg pain

Ulcers

Lower extremity revascularization

the stenoses identified with ultrasound increases the 5-year patency rate up to $93 \% .{ }^{40,41}$ Segmental pressure measurement has not been useful to predict graft failure or the need for revision. ${ }^{42}$ Doppler examination is useful to identify flowreducing lesions before graft failure occurs. Standard surveillance protocols recommend ultrasound evaluation of the graft twice during the first postoperative year, and annually thereafter (Table 16). Early intervention improves long-term patency by $15 \%$ to $20 \%$. Technical failure often causes graft failure in the first month. Intimal hyperplasia and progression of atherosclerotic disease cause graft failure over the ensuring years.

\section{Interpretation}

PSV ratios are determined for grafts in a manner identical to their use in native arteries (Table 17). ${ }^{43,44}$ Exceptions to using distal-to-proximal PSV ratio include cases with a diameter mismatch in the graft or proximal tandem lesions. In these cases a distal PSV can be used instead of the PSV ratio. ${ }^{44}$ Doubling of the velocity PSV ratio indicates significant graft stenosis of greater than $50 \%$, with sensitivity of $95 \%$ and specificity $100 \% .{ }^{45}$

Severe or high-grade lesions warrant intervention. Low velocities indicate poor arterial inflow, proximal stenosis, or large graft diameter. The presence of a parvus et tardus waveform indicates inflow disease or proximal stenosis. In addition, ${ }^{46}$ a PSV less than $45 \mathrm{~cm} / \mathrm{s}$ within a graft indicates that subsequent graft failure is likely to occur. The fundamental criteria of ultrasound evaluation of bypass grafts include: PSV greater than $180 \mathrm{~cm} / \mathrm{s}$ or PSV ratios greater than 2 indicate up to $50 \%$ stenosis; low-flow states $(<45 \mathrm{~cm} / \mathrm{s})$ indicate increased propensity for graft failure; and changes in waveform shape and velocity measurements on serial examinations warrant close follow-up/possible revision. 
Table 15 Diagnostic criteria for peripheral arterial diameter reduction

\begin{tabular}{lclcr}
\hline & Diameter reduction & Waveform & Spectral broadening & PSV distal/PSV proximal \\
\hline Normal & 0 & Triphasic & Absent & +++ No change \\
Mild & $1 \%-19 \%$ & Triphasic & Present & $<2: 1$ \\
Moderate & $20 \%-49 \%$ & Biphasic & Present & $<2: 1$ \\
Severe & $50 \%-99 \%$ & Monophasic & Present & $>2: 1^{*}$ \\
\hline
\end{tabular}

PSV, Peak systolic velocity.

* $>4: 1$ Suggests $>75 \%$ stenosis, $>7: 1$ suggests $>90 \%$ stenosis.

Table 16 Indications for arterial bypass graft ultrasound

- At 3 and 6 mo in the first year after revascularization

- At annual intervals after revascularization after the first year

\section{LOWER EXTREMITY ARTERY ULTRASOUND AFTER PERCUTANEOUS REVASCULARIZATION}

The long-term patency of percutaneous angioplasty and stenting in the lower extremity ranges from 50\% to $85 \% .{ }^{47-50}$ Duplex evaluation is being performed after percutaneous revascularization to detect evidence of stenoses at intervention sites. The concept is that early detection of lesions may assist in identifying individuals who need reintervention. Duplex ultrasound is currently performed after intervention, at recurrence of symptoms, and often annually thereafter. The color and pulsed wave Doppler examination focus on the vessel proximal to the intervention site, at the site, and distal to the site $^{51}$ (Figure 7). Stenosis is evaluated in a manner similar to that used in native arteries, discussed previously (Table 18). A PSV ratio of 2 indicates a stenosis greater than $50 \% .{ }^{44}$

\section{DIAGNOSIS AND MANAGEMENT OF IATROGENIC PSEUDOANEURYSM USING ULTRASOUND}

Increasing interest in endovascular therapy as a primary option for patients with cardiovascular disease has resulted in a greater frequency of vascular complications. Despite the efficacy of these endovascular procedures, complications of vascular access do occur, including hematoma formation, arteriovenous fistulae, infection, atheroemboli, retroperitoneal hemorrhage, native arterial thrombosis, and pseudoaneurysm (PSA). PSA is among the most common iatrogenic vascular complication, and is associated with significant risk of expansion, extrinsic compression on native arteries, rupture, embolization, and infection. A PSA represents a persistent defect throughout all 3 layers of the arterial wall, resulting in extravasation of blood outside of the artery. This extravascular blood is supported by the surrounding soft tissues.
Table 17 Diagnostic criteria for vein graft lesions using peak systolic velocity

- Minimal stenosis $<20 \%$ with PSV ratio $<1.4$ and $<125 \mathrm{~cm} / \mathrm{s}$

- Moderate stenosis of $20 \%$ to $50 \%$ with PSV ratio 1.5 to 2.4 and a PSV $<180 \mathrm{~cm} / \mathrm{s}$

- Severe stenosis $50 \%$ to $75 \%$ with PSV ratio 2.5 to 4 and a PSV $>180 \mathrm{~cm} / \mathrm{s}$

- High-grade stenosis $>75 \%$ with PSV ratio $>4$ and PSV $>$ $300 \mathrm{~cm} / \mathrm{s}$

PSV, Peak systolic velocity.

The key to the diagnosis of PSA remains a high index of suspicion (Table 19). Clinically, any patient who undergoes an arterial puncture for arteriography or endovascular intervention and experiences pain at the access site after the procedure should be considered to be at risk for PSA formation. A pulsatile mass on physical examination and/or the presence of an audible bruit on auscultation over the access site are helpful physical findings. However, many PSAs will not have these findings. Extensive ecchymosis at the access site may also be a clue to the potential presence of a PSA.

PSAs are identified when flow into an extravascular sac is detected (Table 20). In addition to the to-and-fro signal in the neck, the proximal native artery of origin may have a lower resistance spectral waveform when compared with the distal artery (Figure 8).

\section{Treatment Options}

Although serious complications may occur with PSAs, not all PSAs require treatment. There are several options for treatment of PSAs, including observation, surgical repair, and ultrasound-guided compression or thrombin injection (Appendix).

\section{VASCULAR LABORATORY ACCREDITATION}

The laboratory accreditation process provides standards for laboratory procedures, staff experience, and quality assurance. The Intersocietal Commission for the Accreditation of Vascular Laboratories (ICAVL) is one such accrediting agency. ${ }^{19}$ The American College of Radiology also provides laboratory certification (www.acr.org). According to ICAVL, "A 

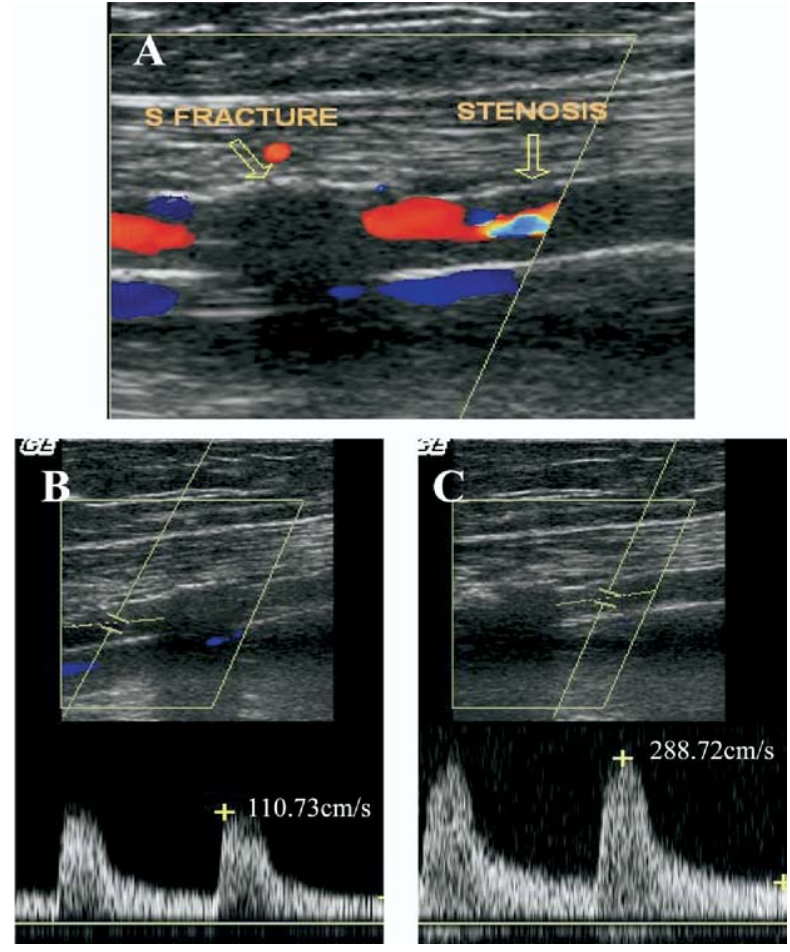

Figure 7 Duplex ultrasound of superficial femoral artery postintervention. A, Color Doppler with laminar flow, followed by loss of color signal at site of stent fracture and complex calcific atherosclerotic plaque, with aliasing distal to stent fracture. B, Spectral Doppler proximal to site of fracture with turbulent flow. C, Peak systolic velocity increases distal to fracture within area of stenosis, suggestive of $50 \%$ to $99 \%$ stenosis.

vascular laboratory is a unit performing noninvasive vascular diagnostic testing under the overall direction of a Medical Director." Their published standards give minimum criteria for physical facilities, ancillary support, and organization of staff. The following areas are highlighted as examples of the requirements for accreditation by this body.

\section{Examination Interpretation, Reports, and Records}

The medical director or a medical staff memberboth of whom must be physicians-should interpret and report on all noninvasive vascular examinations. All reporting must be standardized and include the name of the technologist (sonographer) performing the examination, the date of the examination, clinical indications, description of the test performed, results-including localization and quantification of abnormal findings and comparison with available previous studies, and a signature. The final report must be completed within 2 working days of the examination, except when outstanding clinical information is needed for completion. Critical findings
Table 18 Interpretation criteria for arterial stenosis after percutaneous revascularization

- $P S V>180 \mathrm{~cm} / \mathrm{s}$

- PSV ratios $>2$ indicate significant stenosis

- Changes in waveform shape and velocity measurements on serial examinations warrant close interval follow-up

PSV, Peak systolic velocity.

Table 19 Indications for duplex study for pseudoaneurysm

- Pulsatile mass at arterial access site

- Sudden pain at arterial access site

- Bruit at arterial access site

Table 20 Criteria for diagnosis of pseudoaneurysm sac

- Extravascular arterial sac with flow

- Communication between sac and artery

- Native artery with forward and reverse flow, ie, to and fro

must be reported to the referring physician on the same day as found.

\section{Qualifications of Medical and Technical Personnel}

Medical directors of vascular ultrasound laboratories, either through completion of a formal residency or fellowship or less formal (Continuing Medical Education) training program, must have interpreted, under the supervision of an experienced mentor, 100 cases in each of the areas for which they will be interpreting studies: carotid duplex ultrasound, transcranial Doppler, peripheral arterial physiologic tests, peripheral arterial duplex ultrasound, and venous duplex ultrasound, and 75 visceral vascular duplex ultrasound cases to interpret the latter studies. ${ }^{19}$ Medical directors with at least 3 years of experience in an established practice, but no formal or informal training per se, must have interpreted 300 cases, under the supervision of an experienced mentor, in each of the following areas (for which they will be interpreting cases): carotid duplex ultrasound, transcranial Doppler, peripheral arterial physiologic tests, peripheral arterial duplex ultrasound, and venous duplex ultrasound, and 225 visceral vascular duplex ultrasound cases. ${ }^{19}$ Medical staff must be licensed physicians with similar experience allowing them to participate as interpreters. Beginning in 2006, the American Registry of Diagnostic Medical Sonographers (ARDMS) will be offering certification in vascular laboratory interpretation for physicians, known as the Registered Physician in Vascular Interpretation certification. 

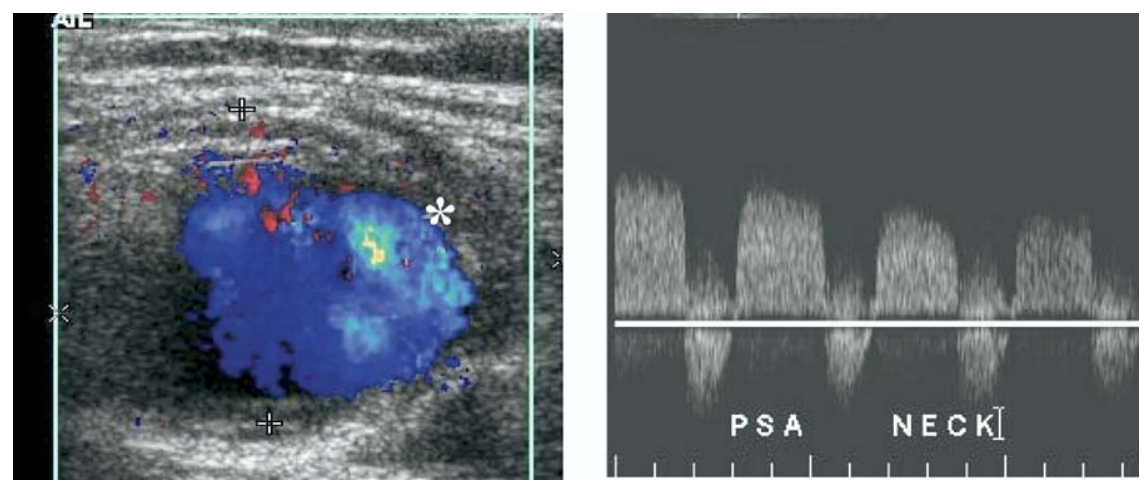

Figure 8 Duplex evidence of pseudoaneurysm (PSA). Left panel, Color Doppler of PSA sac demonstrating thrombus $\left(^{*}\right)$ lining cavity. Color flow is evident within cavity. Right panel, Unique to-and-fro spectral Doppler recording in neck of PSA is diagnostic of PSA.

Technical directors must have experience in performing and interpreting vascular laboratory tests. In addition, technical directors should have had 3 years of vascular testing experience, with the performance of at least 1800 noninvasive vascular examinations with appropriate distribution in testing areas being performed by the laboratory. Since January 2003, all technical directors have been required to have an appropriate credential in vascular testing. Technical staff (sonographers) must have performed 100 cases in each applicable area.

Specific recommendations from ICAVL regarding vascular laboratory operations are available for extracranial cerebrovascular, intracranial cerebrovascular, peripheral arterial, peripheral venous testing, and visceral vascular testing. ${ }^{20-23,25}$ Each module includes information on standards for instrumentation, indications, techniques and components of examination performance, diagnostic criteria, procedure volumes, and quality assurance.

\section{Training of Sonographers}

The ASE has recommended that all sonographers enter and complete training in a program recognized and supported by the ASE, such as those accredited by the Commission on Accreditation of Allied Health Programs, the Joint Review Committee for Cardiovascular Technology, and the Joint Review Committee for Diagnostic Medical Sonography. ${ }^{26}$ Sonographers and physicians can become Registered Vascular Technologists, credentialed by the ARDMS, by successfully completing both the Vascular Physical Principles and Instrumentation and Vascular Technology examinations. Prerequisites to taking the ARDMS examinations vary according to education level and are available on the ARDMS Web site (www.ardms.org/applicants/ prechart.html). Credentialing as a Registered Vascular Specialist is also offered by Cardiovascular Credentialing International (CCI). For details, see the CCI World Wide Web site (www.cci-onlin- e.org). The training qualifications and experience for the sonographers (including the technical director) in a vascular ultrasound laboratory are listed above under "Vascular Laboratory Accreditation."

\section{REIMBURSEMENT: MEDICARE GUIDELINES}

For studies performed in the hospital, Medicare Part A usually covers the technical components of the study, whereas the interpretation is billed under Medicare Part B. The majority of reimbursement falls under Medicare Part B, especially for studies performed in private offices and clinics. The requirement that a vascular laboratory be accredited and/or staff registered and certified to receive Medicare reimbursement for vascular studies is determined by each state's medical carrier. ICAVL provides links to requirements for each state on their Web site (www.icavl.org). Medicare payment policies are enforced through postpayment audits. Laboratory records are reviewed to assure compliance, and providers who violate billing regulations must repay the carrier for past erroneous claims.

\section{REFERENCES}

1. Beller GA, Bonow RO, Fuster V. ACC revised recommendations for training in adult cardiovascular medicine: core cardiology training II (COCATS 2) (revision of the 1995 COCATS training statement). J Am Coll Cardiol 2002;39:1242-6.

2. Philips D, Beach K, Primozich J. Should results of ultrasound Doppler criteria be reported in units of frequency or velocity? Ultrasound Med Biol 1989;15:205-12.

3. Logason K, Barlin T, Jonsson ML, et al. The importance of Doppler angle of insonation on differentiation between 50-69\% and 70-99\% carotid artery stenosis. Eur J Endovasc Surg 2001;21:311-3. 
4. Pellerito J. Color persistence: indicator of hemodynamically significant peripheral arterial stenosis. Radiology 1991; 181:89.

5. Jager K. Non-invasive mapping of lower limb arterial lesions. Ultrasound Med Biol 1985;11:515-21.

6. Rubin J. Power Doppler US: a potentially useful alternative to mean frequency-based color Doppler US. Radiology 1994; 190:853-6

7. Griewing B, Doherty C, Kessler C. Power Doppler ultrasound examination of the intracerebral and extracerebral vasculature. J Neuroimaging 1996;6:32-5.

8. Beckett WW Jr, Davis PC, Hoffman JC Jr. Duplex Doppler sonography of the carotid artery: false-positive results in an artery contralateral to an artery with marked stenosis. AJNR Am J Neuroradiol 1990;11:1049-53.

9. Ringer AJ, et al. Follow-up of stented carotid arteries by Doppler ultrasound. Neurosurgery 2002;51:639-43.

10. Yadav JS, et al. Protected carotid-artery stenting versus endarterectomy in high-risk patients. N Engl J Med 2004;351: 1493-501.

11. Medicare services of Missouri draft policy for non-invasive vascular studies. AC-PN 2003-01, pg. 29, March 2003.

12. Fell G, Breslau P, Knox R. Importance of non-invasive ultrasonic Doppler testing in the evaluation of patients/asymptomatic carotid bruits. Am Heart J 1981;102:221-6.

13. Zwiebel WJ. Duplex sonography of the cerebral arteries: efficacy, limitations, and indications [comment]. AJR Am J Roentgenol 1992;158:29-36.

14. Ackerstaff RG, et al. Ultrasonic duplex scanning of the prevertebral segment of the vertebral artery in patients with cerebral atherosclerosis. Eur J Vasc Surg 1988;2:387-93.

15. Transamerica Occidental Life Insurance Company payment safeguard administrator proposal local medical review policy for the state of California for noninvasive vascular studies.

16. Grant EG, et al. Carotid artery stenosis: gray-scale and Doppler US diagnosis-Society of Radiologists in Ultrasound consensus conference. Radiology 2003;229:340-6.

17. Langlois $Y$, Roederer G, Strandness DJ. Ultrasonic evaluation of carotid bifurcation. Echocardiography 1987;4:141-59.

18. Roederer G, Langlois Y, Jager K. A simple spectral parameter for accurate classification of severe carotid artery disease. Bruit 1989;3:174-8.

19. Mattos MA, Hodgson KJ, Faught W. Carotid endarterectomy without angiography: is color-flow duplex scanning sufficient? Surgery 1994;116:776-83.

20. Dawson D, Zierler R, Strandess DJ. The role of duplex scanning and arteriography before endarterectomy: a prospective study. J Vasc Surg 1993;18:673-83.

21. Hollenberg NK. Medical therapy for renovascular hypertension: a review. Am J Hypertens 1988;1:338-43S.

22. Mailloux LU, et al. Renal vascular disease causing end-stage renal disease, incidence, clinical correlates, and outcomes: a 20-year clinical experience. Am J Kidney Dis 1994;24:622-9.

23. Scoble JE, et al. Atherosclerotic renovascular disease causing renal impairment-a case for treatment. Clin Nephrol 1989;31: 119-22.

24. Gray BH, et al. Clinical benefit of renal artery angioplasty with stenting for the control of recurrent and refractory congestive heart failure. Vasc Med 2002;7:275-9.

25. Eidt J, Fry R, Clagett G. Postoperative follow-up of renal artery reconstruction with duplex ultrasound. J Vasc Surg 1988;8:667-73.
26. Dorros G, Jaff M, Mathiak L. Four-year follow-up of PalmazSchatz stent revascularization as treatment for atherosclerotic renal artery stenosis. Circulation 1998;98:642-7.

27. Harter LP, et al. Ultrasonic evaluation of abdominal aortic thrombus. J Ultrasound Med 1982;1:315-8.

28. Steiner E, et al. Sonographic examination of the abdominal aorta through the left flank: a prospective study. J Ultrasound Med 1986;5:499-502.

29. Yucel EK, et al. Sonographic measurement of abdominal aortic diameter: interobserver variability. J Ultrasound Med 1991;10:681-3.

30. Scott RA, Ashton HA, Kay DN. Abdominal aortic aneurysm in 4237 screened patients: prevalence, development and management over 6 years [comment]. Br J Surg 1991;78:1122-5.

31. Yao ST. Hemodynamic studies in peripheral arterial disease. Br J Surg 1970;57:761-6.

32. Marinelli MR, et al. Noninvasive testing vs clinical evaluation of arterial disease: a prospective study. JAMA 1979;241: 2031-4.

33. Pascarelli EF, Bertrand CA. Comparison of blood pressures in the arms and legs. N Engl J Med 1964;270:693-8.

34. Yao ST, Hobbs JT, Irvine WT. Ankle systolic pressure measurements in arterial disease affecting the lower extremities. Br J Surg 1969;56:676-9.

35. Kohler T. Duplex scanning for diagnosis of aotroiliac and femoropopliteal disease: a prospective study. Circulation 1987; 76:1074-80.

36. Polak J. Determinations of the extent of lower extremity peripheral arterial disease with color-assisted duplex sonography: comparison with angiography. AJR Am J Roentgenol 1990;155:1085-9.

37. Cossman D. Comparison of dye arteriography to arterial mapping with color-flow duplex imaging in the lower extremities. J Vasc Surg 1989;10:522-9.

38. Ranke C, Creutzig A, Alexander K. Duplex scanning of the peripheral arteries: correlation of the peak velocity ratio with angiographic diameter reduction. Ultrasound Med Biol 1992; 18:433-40.

39. Strandness DE Jr, Van Breda A. Surgical management of femoropoliteal occlusive disease. In: Breda SAA, editor. Vascular diseases: Surgical and interventional therapy. New York: Churchill Livingstone; 1994. p. 493-9.

40. Donaldson M, Mannick J, Whittemore A. Causes of primary graft failure after in situ saphenous vein bypass grafting. J Vasc Surg 1992;15:113-20.

41. Bergamini T. Experience with in situ saphenous vein bypasses during 1981 to 1989: determinant factors of long-term patency. J Vasc Surg 1991;13:137-49.

42. Bandyk D. Essentials of graft surveillance. Semin Vasc Surg 1993;6:92-102.

43. Griggs M, Nicolaides A, Wolfe J. Detection and grading of femorodistal vein graft stenosis: duplex velocity measurements compared with angiography. J Vasc Surg 1988;170:661-6.

44. Polak J. Early detection of saphenous vein arterial bypass graft stenosis by color assisted duplex songraphy: a prospective study. AJR Am J Roentgenol 1990;154:857-61.

45. Bandyk D. Nature and management of duplex abnormalities encountered during infrainguinal vein bypass grafting. J Vasc Surg 1996;24:430-8.

46. Bandyk D, Cato R, Towne J. A low velocity predicts failure of femoropoliteal and femorotibial bypass grafts. Surgery 1985; 98:799-809. 
47. Matsi P. Femoropoliteal angioplasty in patients with claudication: primary and secondary patency in 140 limbs with 1-3 year follow-up. Radiology 1994;191:727-33.

48. Murray J, Apthorp L, Wilkins R. Long-segment ( $>$ or +10 $\mathrm{cm})$ femoropoliteal angioplasty: improved technical success and long-term patency. Radiology 1995; 195:158-62.

49. Cejna M. PTA versus Palmaz stent placement in femoropoliteal artery obstructions: a multicenter prospective randomized study. J Vasc Interv Radiol 2001;12:23-31.

50. Soder H. Failure of prolonged dilation to improve long-term patency of femoropoliteal artery angioplasty: results of a prospective trial. J Vasc Interv Radiol 2001;13:361-9.

51. Ahn S. Reporting standards for lower extremity arterial endovascular procedures. J Vasc Surg 1993;17:1103-7.

\section{APPENDIX \\ TECHNIQUE OF CAROTID ARTERY ULTRASOUND}

The first segment of carotid duplex ultrasound examination involves a complete survey of the extracranial carotid arteries in transverse and longitudinal views, an assessment of the anatomy, and the presence or absence of disease and its location. Beginning at the base of either side of the neck, first from the anterolateral and then the posterolateral view, the operator should move the transducer slowly from the level of the clavicle to the level of the jaw. The right CCA is located lateral to the thyroid gland and medial to the internal jugular vein. The ICA is typically located lateral and posterior in the neck, whereas the external carotid artery (ECA) is medial and slightly more anterior. The internal jugular vein is easily compressible in most patients, whereas the CCA is not. In most patients, the origin of the right CCA and the right subclavian artery are easily accessible to the scan head, with slight caudal angulation of the transducer, as they branch from the brachiocephalic artery. Left CCA as proximal as possible at the base of the neck. The origin of the left CCA cannot be identified from this view. Following the CCA to the origin of the ICA and the ECA, the bifurcation is usually found at the level of the cricoid cartilage (level of C3 or C4, but may vary from C1-C6). ICA and ECA should be followed as far in the neck as possible. For assessment of the plaque character and location, the entire CCA, ICA, and ECA should be scanned in B-mode (gray scale) without color.

The longitudinal view is used to correctly place the Doppler sample volume for velocity measurement and for assessment with color Doppler. The CCA is visualized and its entire length is assessed with spectral Doppler from the level of its origin on the right and as low in the neck as possible on the left. Stenosis can be focal and flow patterns can normalize within a short distance beyond the stenosis. For this reason, the sample volume should not be placed in discrete spots but, rather, methodically advanced throughout the length of the vessel using color Doppler for guidance. Representative velocity measurements should be recorded from the most proximal CCA, the mid-CCA, and the distal CCA. The CCA spectral waveform signature normally has greater diastolic flow than the ECA, but less than the ICA. The ICA should be followed as far distally as possible, at least $3 \mathrm{~cm}$ to an area well beyond the disease. Using spectral Doppler, sample volume should be walked throughout the entire ICA, recording velocity samples-PSV and end-diastolic velocity from the proximal, mid, and distal ICA segments. The distal ICA sample should be obtained at least 3 $\mathrm{cm}$ distal to the bifurcation.

\section{CAROTID ARTERY ULTRASOUND: PRACTICAL TIPS}

- ICA and ECA are usually differentiated based on several criteria, as no one criterion by itself is definitive (Table 2). A direct comparison of the waveforms from the two vessels is critical before using the resistance characteristics for identification.

- Physical limitations such as recent neck surgery, radical neck dissection, prior radiation, skin staples, sutures, or dressings require extra ultrasonic gel or standoff to facilitate imaging.

- Use posterior approach for patients who cannot extend the neck because of fixed cervical spine disease, cervical collar, or muscle contractures.

- For patients unable to lay flat, imaging in the seated or standing position can be performed. A low-frequency transducer should be used in patients with a deep artery or thick neck.

- Stenosis may be overestimated in the presence of tortuosity if the sample volume is placed in the outer curvature.

- ICA/CCA ratio may still apply and should be used for identifying the degree of stenosis. In the presence of decreased cardiac output or severe aortic stenosis absolute velocity criteria will not be directly applicable.

- It is important to obtain sample velocities in the maximum area of stenosis, and just proximal and distal to any narrowing to avoid pitfalls in assessment of stenosis.

\section{TECHNIQUE OF RENAL ARTERY DUPLEX ULTRASOUND}

Renal artery duplex ultrasonography requires a vascular technologist who has demonstrated dedication to perfecting the procedure, and state-of-the-art ultrasound equipment. Patients are instructed to fast from midnight before the examination. Each study must be performed in the early morning, and pa- 
tients are instructed to take their morning medications with small sips of liquid. If significant bowel gas is identified, the study is terminated, the patient given simethicone, and the patient is rescheduled for another morning. A low-frequency 2.25- to 3.5$\mathrm{MHz}$ pulsed Doppler transducer is required for adequate deep abdominal imaging. The addition of color imaging will increase the ease with which the renal arteries are identified. The examination is started with the patient supine and in reverse Trendelenburg's position. The aorta is scanned in the longitudinal view from the diaphragm to the aortic bifurcation. The presence of atherosclerotic plaque and aneurysmal dilation is noted. The origin of the celiac, superior, and inferior mesenteric arteries is defined. A Doppler velocity is obtained at the level of the superior mesenteric artery, in the center stream of arterial flow, at a 60-degree angle. This velocity is recorded, and will be used as the denominator for renal-to-aortic velocity ratio calculations.

The transducer is then reoriented into the transverse plane, and the celiac and superior mesenteric arteries are noted arising from the anterior aspect of the aorta. In $75 \%$ of patients, the left renal vein crosses anterior to the abdominal aorta, as it enters the inferior vena cava. These two ultrasound landmarks (origin of the superior mesenteric artery and left renal vein crossing anterior to the aorta) are important for the identification of the right renal artery. The right renal artery arises in an anterior approach, and then courses in posterior fashion as it enters the hilum of the kidney. The left renal artery generally arises inferior to the right, and takes a posterior course. The Doppler cursor, at a 60 -degree angle (or less), is placed within the aorta, and then walked into the ostium of the right renal artery. PSV and peak end-diastolic velocities are obtained in the origin, proximal, mid, and, if possible from this orientation, distal renal artery. The renal artery Doppler waveform has a characteristic low-resistant signal, with significant forward diastolic flow. Similar measurements are obtained from the left renal artery. It is critical that the entire renal artery is visualized, so that a focal stenosis or web (in the case of fibromuscular dysplasia) is not overlooked. Both renal veins are noted to be patent or occluded.

The patient is then placed in the left lateral decubitus position, and the right kidney is visualized from the flank. Three discrete measurements of the pole-to-pole length of the kidney are recorded, in centimeters. Doppler velocities in the cortex, medulla, and hilum of the kidney are recorded at a 0 -degree angle, with a large sample volume, as discrete parenchymal vessels are difficult to identify. The Doppler transducer is then moved slightly anteriorly, and attempts are made to visualize the entire right renal artery from the aorta to the hilum of the right kidney. This banana-peel technique can be particularly helpful in imaging and analyzing the entire renal artery. A limitation of the banana-peel approach on the right is the presence of the overlying inferior vena cava, a problem that does not occur on the contralateral side. This process is then repeated with the patient in the right lateral decubitus position in an effort to interrogate the left kidney and entire left renal artery.

\section{TECHNIQUE OF ABDOMINAL AORTIC ULTRASOUND}

The patient is required to fast before the study. To evaluate an aortoiliac segment, an ultrasound machine with a low-frequency transducer $(2.4 \mathrm{MHz})$ is needed. A midrange transducer $(4-8 \mathrm{MHz})$ is typically used for femoral or popliteal aneurysms. The examination of an aneurysm should be focused on determining the aneurysmal size, shape, location (infrarenal or suprarenal), and distance from other arterial segments.

Ultrasound scanning begins in the supine position. To facilitate accurate measure of its size, 3 sonographic views of the abdominal aorta are usually obtained: transverse plane (A-P diameter and transverse diameters), the sagittal plane (A-P diameter), and coronal plane (longitudinal and transverse diameters). Of note, the transducer should be oriented so that the maximal length of the segment is visualized. The transducer is then rotated 90 degrees to achieve a transverse view. If overlying bowel gas obstructs the aorta from view, patients are placed in the decubitus position and the aorta is visualized by the coronal plane through either flank. The celiac artery may emerge from the aorta and branchi into the common hepatic and splenic arteries. In addition, the right renal artery may be seen emerging from the aorta and traveling under the inferior vena cava.

The most reliable view for measurement of the abdominal aorta is the anterior-posterior image. The lateral view is often the least accurate view because of specular reflection artifact from the vessel wall. Hindrances to an adequate examination include overlying bowel gas and obesity, especially in the region of the proximal neck. ${ }^{30}$

\section{TECHNIQUE OF SEGMENTAL LIMB PRESSURE MEASUREMENT}

Segmental limb pressures are typically measured in conjunction with segmental limb plethysmography (pulse volume recordings). Both procedures are performed using pneumatic cuffs that are appropriately sized to the diameter of the limb segment 
under study and are properly positioned. It is common practice to evaluate the presence of arterial blood flow in the limb distal to the cuff with an appropriate sensor before measuring pulse volume recordings. The patient is initially placed in the supine position for at least 10 minutes before measuring limb pressures. There are several commercially available devices that have pneumatic cuffs with automatic inflation capability. A continuous wave Doppler instrument with a transducer frequency of 4 to $8 \mathrm{MHz}$ is the preferred instrumentation to detect Doppler arterial signals. The pneumatic cuff is initially inflated quickly to a supersystolic value. The cuff is then slowly deflated until a flow signal returns. The cuff pressure at which the flow signal resumes is taken to be the systolic pressure in the arterial segment beneath the cuff. For example, if the cuff is on the high thigh and the sensor is in the popliteal fossa, the measured pressure is reflective of the proximal superficial femoral and profunda femoris arteries, and any collateral arteries, not the popliteal artery. Although published studies indicate that the measured pressure is slightly more accurate when the flow sensor is positioned in close proximity to the cuff, most laboratories for convenience use the Doppler signal from an artery at the ankle for all limb measurements.

Many laboratories use a 4-cuff method where cuffs are positioned as follows: at the high thigh with the upper edge of the cuff positioned at the top most portion of the inner thigh, at the low thigh above the patella, at the calf below the tibial tubercle, and at the ankle above the malleoli. An alternative method involves using only 3 cuffs with a single, relatively wide cuff at the midthigh. Typically, the foot pressure is measured by insonating the posterior tibial and anterior tibial arteries at the ankle level, thus, generating two numbers. Single pressure measurements are made for the calf and the high- and low-thigh levels regardless of the tibial signal selected as the flow indicator. The ankle pressures are used to calculate the ankle-brachial index for each extremity. Each of the ankle pressures is divided by the highest brachial artery pressure.

The lower extremity pressure evaluation begins at the ankle level with a systematic search for arterial signals at ascending levels. Patients who are found to have a normal ankle-brachial index are unlikely to have more proximal disease and an exercise treadmill test or reactive hyperemia test would be necessary to uncover subcritical stenosis. In addition, if the ankle-brachial index is normal and the symptoms are present in the digits of the feet, the toes should be evaluated for the presence of pedal or digital artery obstruction using appropriately sized cuffs.

\section{TECHNIQUE OF PULSE VOLUME PLETHYSMOGRAPHY}

Plethysmography, derived from the Greek "plethysmos," meaning "increase," is used to describe the change in the volume of a limb occurring in response to blood flow into and/or out of that limb. This assessment is made in conjunction with the segmental Doppler pressure measurements. The pulse volume curve is analogous to the arterial pressure pulse. An advantage of using pulse volume recording amplitudes is that they are valid when examining calcified vessels, because the test does not rely on occlusion of the calcified artery as is necessary for segmental pressure measurement. Segmental limb plethysmography (pulse volume recordings) is done using pneumatic cuffs, which are appropriately sized to the diameter of the limb segment under study and are properly positioned. Each cuff is sequentially inflated to a predetermined reference pressure. A cuff pressure of $65 \mathrm{~mm} \mathrm{Hg}$ has been found to achieve surface contact of the cuff to the skin and at the same time impart a reproducible contour characteristic of plethysmography. Plethysmographic waveforms are recorded for each limb segment. Of note, cuff placement may need to be readjusted to ensure that the selected cuff pressure can be achieved within a narrow range of cuff inflation volume. Bilateral testing is considered an integral part of each examination.

\section{TECHNIQUE OF EXERCISE TESTING}

Patients fast for 12 hours before the treadmill test. Before walking on the treadmill, patients are instructed to alert the technician regarding development of any pain. The standard treadmill test is performed at a speed of 2 mile/h and a grade of $12 \%$. The exercise treadmill study is stopped immediately if the patient develops chest pain, or if shortness of breath and dizziness impair the patient's ability to continue. The test is continued otherwise for 5 minutes or until the patient stops secondary to claudication.

After the treadmill is stopped, the patient is immediately brought to a table and placed in the supine position. The ankle pressures are obtained initially, starting with the symptomatic leg. The ankle pressures are repeated every 30 seconds for the first 4 minutes and then every minute for up to 10 minutes. The pressure measurement routine is stopped earlier if the pressure returns to baseline. Of note, the brachial systolic pressure should be recorded immediately at the first set of ankle pressures and at the end of the postexercise ankle pressures. If available, a second technologist should take the 
brachial pressures so as not to delay ankle pressures. Data recorded from the treadmill procedure should include measured ankle pressures, length of time the patient was able to walk, time required for the pressure to return to baseline, nature and location of the patient's symptoms, and reason for discontinuing the test if other claudication or maximum resting time occurs.

\section{TECHNIQUE OF PERIPHERAL ARTERY ULTRASOUND}

Color Doppler is used to determine the presence of laminar or nonlaminar flow states throughout the arterial segments being evaluated. Turbulence, color disturbances such as persistence (continuous forward flow), and aliasing are present at sites of disease. Pulsed (spectral) Doppler sampling is used to further characterize the flow and the degree of stenosis at sites of disturbed color flow. PSV is the primary measure used to quantify disease. Peak velocity measurements are compared between the level of the lesion and proximal arterial segments. Accurate diagnosis of peripheral arterial disease relies in part on technical considerations such as transducer choice and selection of imaging parameters. A 5-MHz linear-array transducer is suitable for examination of the average adult. Higher-frequency probes $(7.5-10 \mathrm{MHz})$ are recommended for small or thin patients and lower-frequency probes $(3-4 \mathrm{MHz})$ for a larger patient or evaluation of deep vessels. The Doppler spectral analysis requires that a small sample volume is used and placed center stream, with the Doppler angle at 60 degrees or less. The sample volume is marched progressively through the length of the arterial system being examined. The color and spectral Doppler are recorded at each new site of interrogation.

\section{TECHNIQUE OF PERIPHERAL ARTERIAL BYPASS GRAFT ULTRASOUND}

The location and type of graft are identified before performing the ultrasound examination. Scanning techniques in the supine patient are similar to those used in native arterial examinations. Color Doppler evaluation of the entire graft is performed with the pulse repetition frequency adjusted to distinguish between laminar flow and regions of aliasing, or persistence. Pulsed Doppler interrogation is guided by the color Doppler findings. The PSV is determined: (1) in the proximal native artery; (2) at the proximal anastomosis; (3) throughout the graft segment; (4) in the distal anastomosis; (5) and in the distal native vessel. Doppler spectra are obtained more frequently at sites of flow disturbance. These velocity measurements are used to diagnose stenosis and to look for change during subsequent examinations.

\section{TECHNIQUE OF EXAMINATION FOR PSA}

PSAs have two components: a sinus tract or neck that extends from the defect in the native arterial wall outside of the artery to the extravascular arterial blood contained by surrounding fibromuscular tissue, and the sac, which is the confined extravascular collection of blood. Color and pulsed wave Doppler evaluation is performed of the femoral arteries and vein. The addition of color to standard gray-scale duplex ultrasonography has made the identification of PSAs highly accurate ${ }^{52}$ and, thus, the diagnostic method of choice. The native artery at the access site is identified, and the extravascular collection of arterial blood is seen, most commonly anterior to the native artery. Posterior PSAs occur most commonly at the bifurcation of the common femoral artery into the superficial and deep femoral artery. The connection between the native artery and the sac, known as the neck of the PSA, is readily identified. Once the neck has been identified using focused Doppler imaging with a narrow sample volume, the Doppler cursor is placed in the neck. If a PSA is present, the pattern of the Doppler waveform obtained will be pathognomonic. The Doppler pattern is a classic to-and-fro waveform, ${ }^{53}$ representing systolic flow out of the native artery into the sac, and diastolic flow back into the native artery from the sac. ${ }^{54}$

\section{REFERENCES}

52. Lacy JH, et al. Pseudoaneurysm: diagnosis with color Doppler ultrasound. J Cardiovase Surg 1990;31:727-30.

53. Abu-Yousef MM, Wiese JA, Shamma AR. The "to-and-fro" sign: duplex Doppler evidence of femoral artery pseudoaneurysm. AJR Am J Roentgenol 1988;150:632-4.

54. Pellerito J, Taylor K. Peripheral arteries. Clin Diagn Ultrasound 1992;27:97-112. 